\title{
ESTUDIO DE LA HERBIVORÍA DE LA PALMA CAMEDOR (CHAMAEDOREA RADICALIS) MART., EN LA SIERRA MADRE ORIENTAL DE TAMAULIPAS, MÉXICO
}

\author{
Gerardo SÁNChez-Ramos ${ }^{* 1}$, Pedro ReYeS-CaSTILlO², \\ Arturo Mora OLIVo' ${ }^{1}$ José Guadalupe MARTínez-Ávalos ${ }^{1}$ \\ IInstituto de Ecología Aplicada, Universidad Autónoma de Tamaulipas Av. División del Golfo \\ No. 356 Col. Libertad C.P. 87020 Cd. Victoria, Tamaulipas, MÉXICO. \\ ${ }^{2}$ Instituto de Ecología, A.C., Departamento Biología de Suelos, Km 2.5 Antigua carretera \\ a Coatepec 351, 91070 Xalapa, Veracruz, MÉXICO. \\ E-mail: gsanchez@uat.edu.mx
}

Sánchez-Ramos, G., P. Reyes-Castillo, A. Mora Olivo \& J. G. Martínez-Ávalos. 2010. Estudio de la herbivoría de la palma camedor (Chamaedorea radicalis) Mart., en la Sierra Madre Oriental de Tamaulipas, México. Acta Zoológica Mexicana (n.s.), 26(1): 153-172.

RESUMEN. Se realizaron análisis de la herbivoría (\% de área foliar consumida día ${ }^{-1}$ ) sobre la palmilla camedor (Chamaedorea radicalis Mart.) durante tres épocas del año: inicio y final de lluvias y época seca en dos diferentes sitios: bosque tropical subcaducifolio y bosque mixto templado en el estado de Tamaulipas, México. Adicionalmente, se realizaron mediciones de algunos parámetros estructurales (altura, cobertura y diámetro del tallo), parámetros físicos de la planta (agua foliar y dureza foliar) y parámetros químicos foliares (nitrógeno foliar y fenoles totales), estos fueron correlacionados con mediciones de herbivoría instantánea. Los resultados muestran mayor consumo foliar al inicio de la época lluviosa $(0.17 \%$ por día), mientras que en la época seca obtuvo el menor registro ( $0.09 \%$ por día). La tasa de herbivoría diaria durante el final de las lluvias fue de $0.13 \%$. Estos valores son más altos de los comúnmente descritos para esos hábitats. Para el bosque tropical las plantas presentaron la mayor altura ( 1.1 veces más), pero con menor cobertura ( 2.1 veces menos). Dentro del bosque tropical esta especie cubre el $2 \%$ del sotobosque, mientras que en el bosque templado cubre el $4 \%$. El contenido de nitrógeno, fenoles totales y agua foliar de las hojas decrecen en tanto que la estación del año progresa, en tanto que la dureza foliar se incrementa. Encontramos que la dureza foliar al finalizar las lluvias y durante la época seca, fue la defensa más efectiva para la herbivoría.

Palabras clave: Insectos herbívoros, bosque mixto templado, bosque tropical caducifolio, dureza foliar, nitrógeno foliar.

Sánchez-Ramos, G., P. Reyes-Castillo, A. Mora Olivo \& J. G. Martínez-Ávalos. 2010. Study of herbivory on the radicalis palm (Chamaedorea radicalis) Mart., in the Sierra Madre Oriental of Tamaulipas, Mexico. Acta Zoológica Mexicana (n.s.), 26(1): 153-172.

ABSTRACT. Rates of insect herbivory (\% of leaf area eaten per day) were measured on the radicalis palm (Chamaedorea radicalis Mart.), during three seasons: early and late rainy season and the middle of the dry season at two different sites: temperate and tropical deciduous forest within the state of

Recibido: 08/10/2009; aceptado: 02/02/2010. 
Tamaulipas, Mexico. In addition, some structural plant parameters (height, cover and diameter stem), physical plant parameters (foliar toughness and water content) and chemical plant parameters (nitrogen and phenols content) were measured, these parameters were correlated with timely (punctual) herbivory measurements. Results from this study show that the early wet season had the most feeding damage $(0.17 \%$ per day); whereas, the dry season had the least damage $(0.09 \%$ per day). The rate of herbivory per day during the middle of the wet season was $0.13 \%$. These values are greater than those commonly reported from such habitats. The highest plants (1.1 times more), but with the least covers corresponded to the tropical deciduous forest. Within the tropical semideciduos forest this specie cover $2 \%$ of understory, whereas to the temperate forest cover $4 \%$. In addition, nitrogen, phenol, and water content of leaves decreased as the season progressed; whereas, leaf toughness increased. We found leaf toughness during the late rainy season and the dry season to be the most effective repellent to herbivory. Key words: Insect herbivory, temperate forest, tropical deciduous forest, leaf toughness, foliar nitrogen.

\section{INTRODUCCIÓN}

La palma camedor (Chamaedorea radicalis) Mart., también conocida como palmilla o palma radicalis (Henley et al. 2000), es una especie del sotobosque que se encuentra en los estados avecindados de Nuevo León, San Luis Potosí y Tamaulipas (Hodel 1992). Se distribuye en la región Huasteca, excepto en el estado de Hidalgo, donde la especie representante es Chamaedorea tepejilote Liebm. (Ponce-Vargas et al. 2006). Sus hojas son de color verde intenso, en un arreglo sobre una vena o nervadura central asemejando una pluma. Son alternas, pinnadas, formando una roseta alrededor del tallo, el cual generalmente es corto. La reproducción es por semillas (Hodel 1992). En México se concentra ca. del 18\% del total de las especies de palmas del planeta, esto es, 95 especies repartidas en 22 géneros (Quero 1994). De ellos, Chamaedorea, género que sólo existe en el continente americano y que en total cuenta con más de 130 especies, es el mayor, con 50 especies, de las cuales 14 son endémicas (CCA 2003). Esta riqueza posiciona a México como el país con mayor número de especies y de endemismos de Chamaedorea y, probablemente uno de los dos centros de diversificación del género (Hodel 1992).

La importancia de esta especie radica en la comercialización de su follaje, que es cosechado manualmente por los campesinos dentro de sus poblaciones naturales (Trejo 1992). Sin embargo, para obtener un óptimo valor comercial las plantas deben estar en su etapa de madurez fisiológica (hojas de $60 \mathrm{~cm}$ con el peciolo $\geq 15 \mathrm{~cm}$ ), intactas y sin daño por herbívoros (Trejo 1992). La colecta extensiva del género Chamaedorea ha ocurrido históricamente en 10 estados de la República Mexicana generando una impactante tasa extractiva, calculada en un millón de hojas diarias y más de $200 \mathrm{~kg}$ de semillas diariamente (Oyama 1987). Su demanda obedece a lo que se denomina en el mercado internacional como "Floricultura y horticultura ambiental" (CCA 2003). Este creciente mercado de follaje resulta por demás interesante e impactante para la economía local, nacional y mundial. Ocurre a través de un proceso itinerante, va desde la extracción y/o producción, hasta la 
comercialización y el destino final (generalmente Estados Unidos de Norteamérica o Europa). La producción alcanzada por ocho estados tan solo en 1999 fue de 2,000 toneladas que generaron 20 millones de pesos. Los márgenes de comercialización para cada uno de los agentes de la cadena comercial se incrementan. El pago que recibe el colector-productor por un ramillete compuesto de 144 hojas (gruesa) es sólo de 12 pesos, llegando al minorista en México o mayorista en Estados Unidos con un costo de 180 pesos, esto es $c a$. 7\% del precio final (CCA 2003). En Tamaulipas, esta situación presiona fuertemente a las poblaciones naturales, ubicadas algunas en zonas de reservas ecológicas (i.e. Reserva de la Biosfera El Cielo, Área Natural Protegida Altas Cumbres). Debido a esta presión extractiva C. radicalis ha sido catalogada como especie amenazada (Vovides 1981), como especie vulnerable (Hodel 1992) y, como especie protección especial por la NOM-059-ECOL-2001.

Una de las interacciones bióticas de mayor impacto en las comunidades naturales es la herbivoría (Crawley 1983; Dirzo 1984). Se estima que aproximadamente el 10\% de la productividad primaria terrestre se mueve en dirección de los herbívoros (Coley et al. 1985). Estos también pueden influir significativamente en la riqueza de especies de plantas, la abundancia relativa de éstas, y ser componentes causales de la heterogeneidad especial (Crawley 1983). El daño foliar que causan los herbívoros reduce el proceso adaptativo de las plantas, ya que, al ser consumida el área fotosintética, altera la absorción radicular de nutrientes (Harper 1977). La herbivoría también causa cambios internos en la distribución de los recursos (Bazzaz et al. 1987), estimulando la compensación de la planta (McNaughton 1983; Trumble et al. 1993). La respuesta ante el ataque de los herbívoros, dependerá de la disponibilidad de nutrientes y la edad de la misma (Hunter \& Schultz 1995; Karban \& Baldwin 1997).

Se considera al fenómeno de la herbivoría, como una importante y decisiva fuerza de selección en el proceso evolutivo de las plantas (Marquis 1987) y juega un papel determinante en la dinámica foliar. La variación intraespecífica del daño por herbivoría afecta el éxito adaptativo de las plantas. Los herbívoros afectan significativamente la riqueza, la heterogeneidad, el ciclaje de nutrientes y la abundancia relativa de las especies de las cuales se alimentan (Crawley 1983). Sin embargo, el estudio sobre la interacción planta-insecto revela la existencia de excepcionales capacidades adaptativas de las plantas para eludir, repeler, retardar y restringir la infestación o el daño causado por insectos. Adicionalmente, así como algunas plantas exhiben tolerancia, otras exhiben una recuperación posterior al daño (Crawley 1983), este hecho se expresa por los mecanismos de tolerancia que exhiben las plantas. Al identificar estos mecanismos de tolerancia al daño por herbívoros facilita los intentos de entender el papel de la tolerancia en la dinámica evolutiva tanto de plantas como de herbívoros (Tiffin 2000).

En términos generales, el daño foliar por herbivoría puede ser hasta diez veces más en hojas jóvenes que en hojas maduras, incluso dentro de la misma planta (Coley 
1990). En adición, la herbivoría puede ser tres veces más en especies pioneras, respecto a especies tolerantes a la sombra (Coley 1983), y más de dos veces en la época lluviosa respecto a la época seca. Así mismo, la herbivoría constituye el factor más importante en el aclareo de la vegetación en un bosque maduro (Coley 1990). Un estudio previo (Sánchez-Ramos 2002), llevado a cabo dentro de la comunidad vegetal, propia del bosque mesófilo, mostró que la herbivoría en este ecosistema varió de 6-9\% dependiendo del estado de madurez del bosque mesófilo (mayor en los claros de regeneración natural que el bosque maduro).

Se ha detectado que los insectos son el agente causal de la herbivoría, en una variedad de hábitats que van desde bosques templados a tropicales (Crawley 1983, Dirzo 1987; Janzen 1981) y de estos, las larvas de Lepidoptera junto con algunos Coleópteros (Chrysomelidae) y hormigas defoliadoras (Attini), constituyen en forma colectiva los más efectivos folívoros (Dirzo \& Domínguez 1995).

Los estudios sobre las interacciones entre herbívoros y plantas de las comunidades vegetales tropicales y subtropicales se han ido incrementando marcadamente en los últimos años (Coley 1983; Dirzo 1984; Janzen \& Waterman 1984; Marquis 1984; entre otros). Dentro de estos herbívoros, se han reconocido ocho especies de crisomélidos del género Calyptocephala los cuales son los principales herbívoros asociados al género Chamaedorea desde México hasta Brasil (Oyama \& Dirzo 1991). Los herbívoros pueden afectar negativamente uno o más componentes de la adecuación de las plantas (e.g. crecimiento, sobrevivencia, reproducción), y han sido considerados como importantes agentes selectivos en la evolución de las mismas (Ehrlich \& Raven 1964; Feeny 1976, Marquis 1984; Rhoades \& Cates 1976; Whitman \& Mooper 1985). Se le ha dado una importancia especial a las diferencias interespecíficas de la herbivoría, específicamente de dioicismo en algunas comunidades neotropicales (Oyama \& Dirzo 1991).

Los niveles de daño por estos herbívoros ocurren a niveles tan básicos como es la hoja dentro del mismo individuo, respecto a su edad (desde 10 veces mayor las tasas de consumo en las hojas jóvenes respecto a las maduras (Coley 1990). Otros trabajos aluden a estudios particulares sobre especies (dioicas), tal es el caso de Neea psychotroides sobre la variación intersexual en la herbivoría de flores y formación de agallas (Wofle 1997). Con respecto a lo anterior, destaca un estudio sobre la diferenciación sexual del daño por herbivoría y la respuesta química de Chamaedorea radicalis, llevado a cabo en la región de Gómez Farías por González et al. (2003). En este estudio los autores obtuvieron diferencia en el consumo foliar, ya que las plantas hembra presentaron $47.5 \%$ mayor consumo foliar, con respecto a las plantas macho. En adición a lo anterior, las plantas hembra en la época lluviosa exhibieron $17.54 \%$ más daño respecto a la época seca, en tanto, las plantas macho muestran $1.3 \%$ de mayor herbivoría en la época lluviosa que en la época seca. Las 
plantas macho registraron un incremento en la herbivoría de $7.8 \%$, mientras que las plantas hembra tuvieron un incremento de 9\% (González et al. 2003).

Un enorme contingente de sustancias químicas, que al parecer no intervienen directamente en las funciones vitales primarias (fotosíntesis, respiración, etc.), han sido detectados en los tejidos vegetales de una gran cantidad de especies, estos compuestos han llegado a nosotros con el nombre de compuestos o metabolitos secundarios (los taninos y los fenoles, entre otros), su función esta asociada a la defensa de la planta, en particular, contra el ataque de los animales herbívoros entre ellos los insectos (Dirzo 1986). Estos son producidos por las plantas (metabolitos secundarios, derivados del metabolismo primario) y han sido implicados como agentes defensivos en algunas interacciones bióticas (Bell 1974; Deverall 1977). Algunos estudios resaltan la asociación existente entre el efecto de la herbivoría con estos compuestos, pues ya que en la mayoría de los casos el consumo de follaje reduce substancialmente la adaptación de las plantas, los compuestos secundarios actúan mitigando o reduciendo los ataques producidos por los herbívoros (Coley 1981, 1983; Coley et al. 1985; Coley \& Aide 1991; Crankshaw \& Langenheim 1981; Marquis 1984; Waterman \& McKey 1989).

Dentro de estos compuestos, los fenoles han sido considerados como una de las mayores defensas contra los defoliadores (Rhoades 1979; Whittaker \& Feeny 1971) y es posible encontrarlos en el follaje de todas las plantas terrestres superiores (Harborne 1988). El término fenólico se ha utilizado para definir aquellas substancias que poseen uno o más hidroxilos $(\mathrm{OH})$, unidos a un anillo aromático (Waterman \& Mole 1994). Las investigaciones efectuadas con fenoles fueron básicas para desarrollar la teoría de la apariencia (Feeny 1976; Rhoades \& Cates 1976), así como de las ideas que explican y sustentan la distribución de los recursos (Coley et al. 1985). Las apreciaciones que actualmente tenemos sobre coevolución, defensas inducidas en plantas y dinámica cíclica planta-herbívoro, también han sido dependientes del estudio de los compuestos fenólicos (Schultz \& Baldwin 1982).

Algunos estudios revelan que la cuantificación fenólica es un método efectivo para evaluar el nivel defensivo químico de la planta en respuesta al daño de la herbivoría (Coley 1983; Coley \& Aide 1991; Gershenzon 1984; Waterman \& Mole 1994; Zucker 1983).

Considerando que la herbivoría es un fenómeno presente en los ecosistemas y que las plantas presentan estrategias defensiva para lograr su adecuación direccionamos el presente estudio con la finalidad de atender las siguientes interrogantes: ¿De qué magnitud es la densidad de $C$. radicalis en dos diferentes tipos de vegetación donde existen poblaciones naturales (bosque tropical subcaducifolio y bosque templado)? ¿Cuál es el nivel de herbivoría en ambos tipos de vegetación? ¿Cómo ocurre la variación estacional de la herbivoría en estos tipos de vegetación? y, finalmente 
¿Cuáles son, cómo operan y cómo se relacionan los atributos que favorecen o disuaden a los herbívoros con $C$. radicalis?

\section{MATERIAL Y MÉTODOS}

Área de estudio. El presente estudio se llevó a cabo en dos localidades del estado de Tamaulipas, México. Ambos sitios corresponden a dos tipos de vegetación contrastantes y están representados por tres estratos de vegetación: 1) Arbóreo, 2) arbustivo, y 3) herbáceo. El Sitio 1 corresponde a un bosque tropical subcaducifolio y el Sitio 2 representa a un bosque templado mixto, que consiste en una zona de ecotonía del bosque tropical, el bosque mesófilo de montaña y encinares (véase Cuadro 1).

Parámetros alométricos de la planta. Dentro de cada sitio fueron seleccionados al azar 10 transectos de $2 \mathrm{~m}$ x $50 \mathrm{~m}\left(100 \mathrm{~m}^{2}\right)$ de acuerdo con Gentry (1982), éstos fueron marcados permanentemente con cinta fosforescente $\left(1,000 \mathrm{~m}^{2}=0.1 \mathrm{ha}\right)$. Dentro de cada transecto se registraron todas las plantas de $C$. radicalis considerando los siguientes parámetros foliares: la altura (tomando el ápice foliar mayor desde la base del tallo, en metros), el diámetro (considerado a $5.0 \mathrm{~cm}$ de la base del tallo, con un vernier digital), y la cobertura (tomando el área foliar total, en $\mathrm{m}^{2}$ ). El efecto causado por daño antropogénico (extracción de hojas) también fue considerado. Este tipo de daño es fácilmente distinguible (Sánchez-Ramos, observ. pers.).

Tasa de herbivoría. El daño foliar fue medido durante las siguientes épocas: 1) a mitad de la época lluviosa (7 Julio 1999), 2) a final de la época lluviosa (7 Octubre 1999), y 3) a mitad de la época seca (7 Febrero 2000) (véase Cuadro 2). De un total de 25 plantas seleccionadas al azar en cada sitio, se consideraron tres hojas jóvenes por planta para llevar a cabo el análisis foliar, (3 hojas x 25 plantas x 2 sitios x 3 épocas; $\mathrm{n}=450$ mediciones foliares). Las hojas fueron seleccionadas a inicios de la época lluviosa (9 mayo 1999). El porcentaje de área foliar consumida fue registrada con un medidor de índice foliar (C1-202 Leaf Area Meter-CID, Inc.). La tasa de herbivoría (TH), se llevó a cabo siguiendo la fórmula de Dirzo (1987), validada por Filip et al. (1995), Coley (1983) y, Sánchez-Ramos (2002):

$$
\mathrm{TH}=\frac{\mathrm{AFC}^{1}-\mathrm{TAF} \times 100}{\mathrm{t}}
$$

Donde, $\mathrm{AFC}_{1}=$ corresponde al área foliar consumida en el tiempo 1 (calculada de la sustracción de TAF en el tiempo 0), TAF= es el total del área foliar (es el área foliar en el tiempo 0), y t = es el número de días considerado entre cada análisis. Entonces, fueron acumulados: $0,59,151$, y 274 días entre cada análisis respectivamente, para construir con esto tres tasas de herbivoría. El daño foliar causado por insectos fue diagnosticado por las cicatrices foliares (ver Dirzo 1987; Sánchez-Ramos et al. 1999; Sánchez-Ramos \& Reyes-Castillo 2006). 
Parámetros foliares. Para analizar la dureza foliar se colectaron y midieron 20 hojas in situ de cada una de las siguientes épocas: 1) inicio de la época lluviosa, 2) final de la época lluviosa, y 3) final de la época seca, quedando el modelo representado como sigue: 20 hojas x 2 sitios x 3 épocas; $n=120$ hojas. Para determinar este parámetro se utilizó un penetrómetro foliar (Pocket Penetrometer) (Dirzo 1987), con él se midió la presión necesaria ejercida para penetrar la hoja en $\mathrm{g} / \mathrm{cm}^{2}$, de acuerdo al método descrito por Feeny (1970). Adicionalmente, para determinar el contenido de agua foliar fueron seleccionadas 20 hojas adicionales dentro de cada sitio y de cada época. Este follaje fue pesado utilizando una balanza analítica ACCULAB (V-200). Las hojas fueron secadas en una estufa de convección forzada a $90^{\circ} \mathrm{C}$ por 30 minutos, el contenido de agua foliar fue medido siguiendo el método de Turner (1981) utilizando la siguiente fórmula:

$$
\text { Contenido de agua foliar }(\%)=\frac{\text { peso fresco }- \text { peso seco }}{\text { peso saturado }- \text { peso seco }} \times 100
$$

El contenido de nitrógeno foliar fue determinado de 20 hojas seleccionadas al azar de ambos sitios y de cada época, quedando el modelo de la siguiente forma: 10 hojas x 2 sitios x 3 épocas; $n=60$ hojas, de acuerdo al método de Microkjeldhal (Technicon Industrial Systems 1977).

Para determinar el contenido total de fenoles se utilizó la técnica de FolinCiocalteu (Sigma-2790) (Waterman \& Mole 1994). De manera similar fueron obtenidas 10 muestras foliares (hojas) de cada sitio en cada época y fueron analizadas, (10 hojas x 2 sitios x 3 épocas; $n=60$ hojas). Para analizar el contenido de fenoles se obtuvo de cada muestra foliar $1 \mathrm{~g}$ de peso fresco, utilizando una balanza electrónica (OHAUS; CT-600) que posteriormente fue deshidratado y macerado. De cada muestra deshidratada y mecerada, se tomó $0.05 \mathrm{~g}$ de peso agregándosele $10 \mathrm{ml}$ de etanol al 80\%, centrifugando a 3000 rpm durante 10 min. Posteriormente, se tomó el sobrenadante, agregándole $10 \mathrm{ml}$ de etanol al $80 \%$ y repitiendo la centrifugación. Después, se tomó del sobrenadante $1 \mathrm{ml}$, a éste se le agregaron $7 \mathrm{ml}$ de agua destilada y se agitó en vortex. Posterior a la agitación, a la muestra se le agregó $0.5 \mathrm{ml}$ del reactivo Folin-Cicolteau agitando nuevamente en vortex. Finalmente, la muestra se dejó reposar por $3 \mathrm{~min}$, agregando $1 \mathrm{ml} \mathrm{de} \mathrm{Na}_{2} \mathrm{CO}_{3}$ (20\%), agitando nuevamente en vortex. Esta mezcla final se reposó por $1 \mathrm{~h}$, para leer finalmente en un espectrofotómetro (M-230), ultravioleta (UV) visible, calibrado a 750 nanómetros (1 $\mathrm{nm}=10^{-9} \mathrm{~m}$ ). La solución estándar de referencia utilizada fue ácido tánico a 100 ppm para generar los puntos de la curva patrón. Las lecturas obtenidas fueron registradas en partes por millón (ppm), y los resultados se expresan en $\mathrm{mg} / \mathrm{g}$ de peso seco (Waterman \& Mole 1994). 
Sánchez-Ramos et al.: Estudio de la herbivoría de Chamaedorea radicalis

Cuadro 1. Sitios de estudio, ubicación, características abióticas y especies representativas. Ambos sitios corresponden a la Reserva de la Biosfera El Cielo, Tamaulipas (Tomado de Mora et al., 1997).

\section{\begin{tabular}{lll}
\hline Características & Sitio 1 (La Libertad) & Sitio 2 (Alta Cima)
\end{tabular}}

\begin{tabular}{lcc}
\hline Posición geográfica & $99^{\circ} 09^{\prime} 00^{\prime} \mathrm{W}^{\mathrm{W}} ; 23^{\circ} 08^{\prime} 01^{\prime \prime} \mathrm{N}$ & $99^{\circ} 11^{\prime} 00^{\prime} \mathrm{W}^{\mathrm{W}} ; 23^{\circ} 033^{\prime} 06^{\prime} \mathrm{N}^{\mathrm{N}}$ \\
Tipo de vegetación & Bosque tropical subcaducifolio & Bosque mixto templado \\
Altitud (m snm) & 140 & 920 \\
Precipitación $(\mathrm{mm})$ & 1,289 & 2,522 \\
Temperatura $\left({ }^{\circ} \mathrm{C}\right)$ & 25.5 & 13.8
\end{tabular}

Especies características: Pseudobombax ellipticum (Bombacaceae), Mirandaceltis monoica (Ulmaceae),

Estrato arbóreo Neopringlea integrifolia (Sapindaceae),

Bursera simaruba (Burseraceae)

Estrato arbustivo

Estrato herbáceo
Randia obcordata (Rubiaceae),

Ocotea tampicensis (Lauraceae),

Croton niveus (Euphorbiaceae),

Bauhinia divaricata (Fabaceae),

Jacobinia incana (Acanthacae)

Chamaedorea radicalis (Arecaceae),

(Cyperaceae),

Syngonium podophyllum (Arecaceae)

Anonna globiflora (Anonnaceae),
Trichilia havanensis (Meliaceae),

Garrya laurifolia (Garryaceae),

Acacia angustissima (Fabaceae)

Eugenia capuli (Myrtaceae), Rapanea myricoides (Myrsinaceae), Exostema mexicana (Rubiaceae),

Decatropis bicolor (Rutaceae),

Rhynchospora aff. aristata

Chamaedorea radicalis (Arecaceae),

Leersia monandra (Poaceae)

Mirabilis jalapa

Cuadro 2. Tasas de herbivoría, épocas analizadas y tiempo acumulado entre épocas para los sitios de estudio: Sitio 1, La Libertad (bosque tropical subcaducifolio) y Sitio 2, Alta Cima (bosque mixto templado).

\begin{tabular}{lcccc}
\hline Análisis de la herbivoría & $\begin{array}{c}\text { Inicio de } \\
\text { época lluviosa }\end{array}$ & $\begin{array}{c}\text { Mediados de } \\
\text { época lluviosa }\end{array}$ & $\begin{array}{c}\text { Final de } \\
\text { época lluviosa }\end{array}$ & Época seca \\
\hline & (9 Mayo 1999) & (7 Julio 1999) & (7 Octubre 1999) & (7 Febrero 2000) \\
\hline Análisis de herbivoría & 1 & 2 & 3 & 4 \\
Tasa de herbivoría & - & Primera & Segunda & Tercera \\
Tiempo (días) acumulado & 0 & 59 & 151 & 274 \\
\hline
\end{tabular}


Como un experimento adicional, y de manera complementaria para correlacionar los parámetros foliares (dureza, contenido de agua foliar, nitrógeno foliar y contenido fenólico) con la herbivoría (Área Foliar Consumida), se seleccionaron 120 hojas adicionales de los sitios, con la finalidad de evaluar cada parámetro en cada época, pero considerando a ambos sitios como una sola muestra, quedando el método de la siguiente manera: 40 hojas x 3 épocas; $n=120$ hojas por cada parámetro foliar (lo anterior en virtud de que había que destruir el follaje para su análisis). Los resultados fueron analizados utilizando un análisis de correlación $\left(r^{2}\right)$, de manera individual en cada parámetro. En todos los casos la variable dependiente $(y)$ fue la herbivoría puntual, y como factores se consideraron los parámetros foliares.

Análisis estadístico. Para determinar posibles diferencias entre la estructura alométrica del follaje entre sitios (i.e., altura, diámetro, cobertura, y número de hojas), se realizó un análisis Kruskal-Wallis (Zar 1984). Para determinar las diferencias en las tasas de herbivoría se llevó a cabo un Análisis de Varianza (ANOVA de medidas repetidas), considerando el porcentaje de daño foliar como la variable dependiente con los sitios y las épocas como factores de dos y tres niveles. En adición se llevó a cabo un Análisis de Varianza (ANOVA) de dos vías para determinar posibles diferencias estadísticas entre parámetros foliares (i.e., contenido de agua, nitrógeno, dureza y fenoles totales) considerando a los sitios y las épocas como los factores. Los resultados de la herbivoría y contenido de agua (porcentajes) fueron transformados en arcoseno para cumplir con los supuestos de normalidad y homocedasticidad. Los resultados se presentan con los datos en su escala original. Los análisis fueron analizados con el programa STATISTICA 8.0.

\section{RESULTADOS}

Parámetros alométricos de la planta. La densidad (número de individuos) de $C$. radicalis, mostró una marginal diferencia entre los sitios. El bosque tropical subcaducifolio mostró un total de 190 plantas, en tanto que el bosque mixto templado presentó 176 (14 menos). Extrapolando estos datos pudiera potencialmente tenerse 1900 plantas/ha para el bosque tropical subcaducifolio y 1760 plantas/ha para el bosque mixto templado. Sin embargo, el número de hojas por planta fue menor 1.3 veces en el bosque tropical (Sitio 1), respecto al bosque mixto templado (Sitio 2).

Se detectaron diferencias estadísticas en los parámetros estructurales de $C$. radicalis entre los dos sitios analizados (Análisis de Kruskal-Wallis) para la altura $\left(H_{1,338}=7.9 ; P=0.004\right)$, el diámetro del tallo $\left(H_{1,338}=11.9 ; P=0.0006\right)$, y la cobertura $\left(H_{1,338}=29.2 ; P=0.0004\right)$ (Fig. 1$)$. La altura promedio (media \pm 1 D.E.) de las plantas en el Sitio 1 (La Libertad) fue 1.1 veces mayor con $0.63 \mathrm{~m}( \pm 0.09)$, respecto al Sitio 2 (Alta Cima) con $0.58 \mathrm{~m}$ ( \pm 0.3 ); el diámetro del tallo en La Libertad también exhibió mayor tamaño 1.2 veces con $1.01 \mathrm{~cm}( \pm 0.1)$, respecto a Alta Cima (0.87 $\mathrm{cm} \pm 0.09)$. En cambio la cobertura promedio de las plantas fue 
mayor en Alta Cima (bosque mixto templado) con $0.21 \mathrm{~m}^{2}( \pm 0.1)$, mientras el bosque tropical subcaducifolio (Sitio 1) mostró 1.2 veces menor tamaño $\left(0.1 \mathrm{~m}^{2} \pm\right.$ 0.1) (Fig. 1). En el bosque tropical subcaducifolio $C$. radicalis cubre $c a .2 .0 \%$ del sotobosque analizado. En este sitio pudimos determinar que $c a .55 .0 \%$ de las hojas (260) fueron extraídas por los campesinos para su comercialización. En cambio para el bosque mixto templado (Alta Cima), $C$. radicalis cubre $c a .4 .0 \%$ del sotobosque y con una extracción para su comercialización de ca. 33.0\% de las hojas (178).

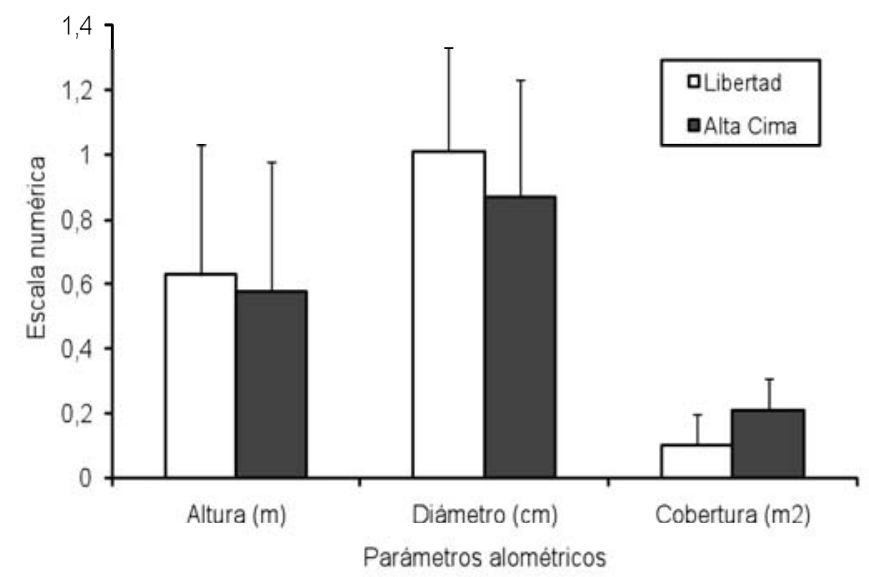

Figura 1. Parámetros alométricos estructurales de Chamaedorea radicalis en dos sitios de estudio: Sitio 1, La Libertad (bosque tropical subcaducifolio) y Sitio 2, Alta Cima (bosque mixto templado). Los valores están expresados en media ( \pm 1 D.E.).

Tasas de herbivoría. El consumo foliar en los dos sitios corresponde a insectos y ácaros (aproximadamente $97 \%$ de un total de 450 plantas), el cual fue determinado visualmente detectando las cicatrices foliares en las pinnas y peciolo de las hojas. Esto es muy similar a lo que presentan las especies tropicales en la región de Los Tuxtlas, México (véase Dirzo 1987) y similar a lo que en un estudio detectó dentro del bosque mesófilo, adyacente a los sitios analizados en la presente investigación en Tamaulipas, México (Sánchez-Ramos 2002), daños causados principalmente por lepidópteros y coleópteros (Sánchez-Ramos et al. 1999). Las tasas de herbivoría fueron consistentes entre las épocas dentro de los sitios analizados (Fig. 2). Las diferencias encontradas fueron significativas (ANOVA de medidas repetidas) entre sitios $\left(F_{1,144}=10.69 ; P=0.001\right.$ ); entre épocas (edad de la hoja) con $F_{2,144}=260.10 ; F<0.001$ ), pero no para la interacción entre época y sitio $\left(F_{1,144}=1.787 ; P=0.48\right)$. El efecto de la acumulación de herbivoría (\% área consumida de la lámina foliar), fue para el Sitio 1 (bosque tropical subcaducifolio) con $23.16 \%$ del área total consumida, esto fue 1.1 veces más que para el Sitio 2 (bosque mixto templado) que presentó $20.15 \%$ (Fig. 3). 


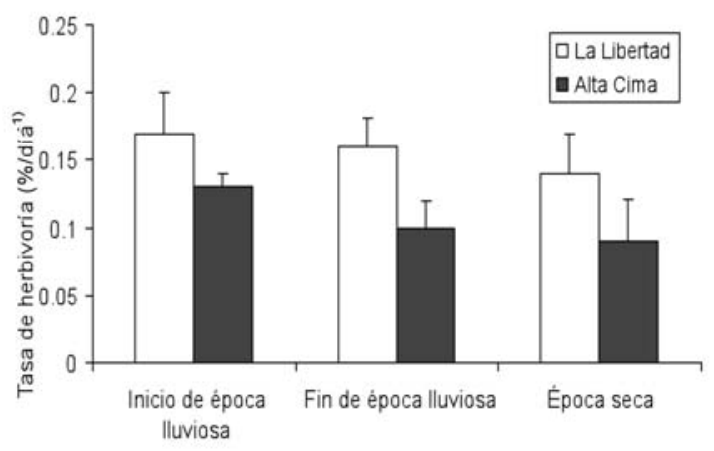

Figura 2. Herbivoría (\% área foliar consumida por día) de Chamaedorea radicalis en dos sitios de estudio: Sitio 1, La Libertad (bosque tropical subcaducifolio) y Sitio 2, Alta Cima (bosque mixto templado). Los valores están expresados en media ( \pm 1 D.E.).

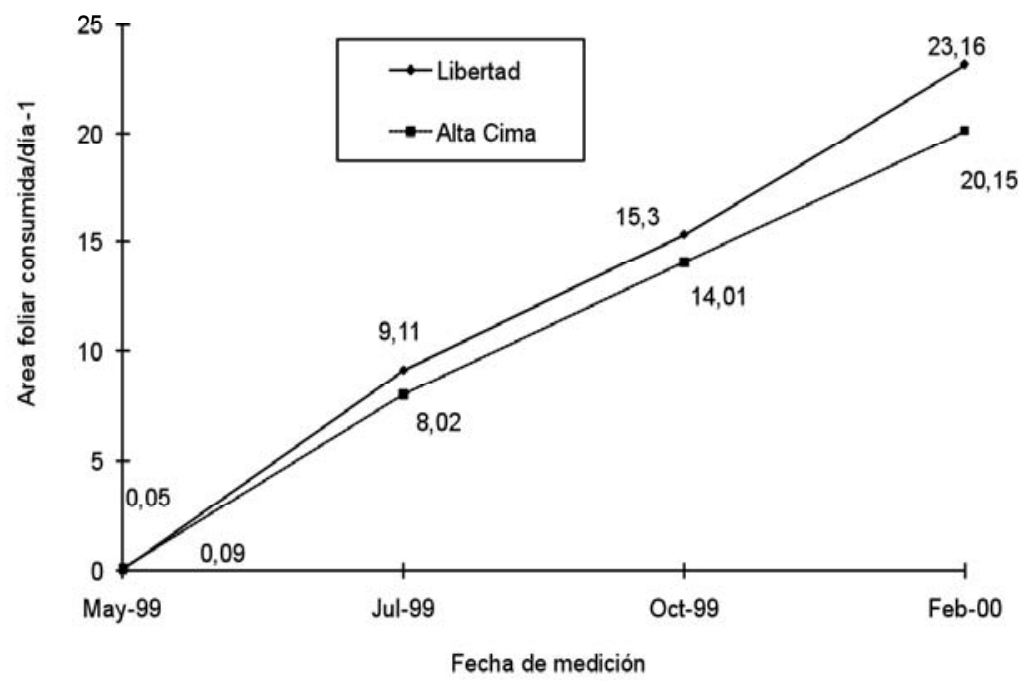

Figure 3. Herbivoría total de Chamaedorea radicalis en dos sitios de estudio: Sitio 1, La Libertad (bosque tropical subcaducifolio) y Sitio 2, Alta Cima (bosque mixto templado). Los valores representan el porcentaje anual acumulado. 
Parámetros foliares. La dureza foliar mostró un incremento en las épocas analizadas para ambos sitios. Para el bosque tropical subcaducifolio (Sitio 1) este incremento fue 3.5 veces al final del análisis (época seca) y de 3.9 veces mayor para el bosque mixto templado (Cuadro 3), resultando estadísticamente significativo $\left(F_{2,114}=\right.$ $1,887.2 ; P<0.001)$. Sin embargo, este no resultó significativo entre sitios $\left(F_{1,114}=\right.$ $0.9 ; P=0.348)$ ni en la interacción época-sitio $\left(F_{2,114}=2.8 ; P=0.066\right)$. La dureza foliar acusó una correlación negativa con la herbivoría al final de la época lluviosa $\left(r^{2}=-0.72 ; P<0.001\right)$ y para la época seca $\left(r^{2}=-0.085 ; P<0.001\right)$ (Cuadro 4$)$.

Cuadro 3. Parámetros foliares (+ 1 D.E.) de Chamaedorea radicalis para los sitios de estudio: Sitio 1, La Libertad (bosque tropical subcaducifolio) y Sitio 2, Alta Cima (bosque mixto templado) en tres épocas del año, Tamaulipas, México.

\begin{tabular}{lcccccc}
\hline Parámetros foliares & \multicolumn{2}{c}{ Inicio de época lluviosa } & \multicolumn{2}{c}{ Final de época lluviosa } & \multicolumn{2}{c}{ Época seca } \\
\hline & $\begin{array}{c}\text { Sitio 1 } \\
\text { Libertad }\end{array}$ & $\begin{array}{c}\text { Sitio } 2 \\
\text { Alta Cima }\end{array}$ & $\begin{array}{c}\text { Sitio 1 } \\
\text { Libertad }\end{array}$ & $\begin{array}{c}\text { Sitio } 2 \\
\text { Alta Cima }\end{array}$ & $\begin{array}{c}\text { Sitio 1 } \\
\text { Libertad }\end{array}$ & $\begin{array}{c}\text { Sitio } 2 \\
\text { Alta Cima }\end{array}$ \\
\hline Tasa (\%) herbivoría & 15.35 & 13.44 & 4.09 & 3.96 & 2.86 & 2.24 \\
Agua (\%) & $34.7 \pm 8.4$ & $30.5 \pm 7.4$ & $26.1 \pm 13.1$ & $21.8 \pm 12.8$ & $21.3 \pm 11.3$ & $17 \pm 9.7$ \\
Nitrógeno foliar (\%) & $3.9 \pm 1.1$ & $3.5 \pm 1.4$ & $2.4 \pm 1.9$ & $2.1 \pm 1.2$ & $1.9 \pm 0.9$ & $1.6 \pm 0.1$ \\
Dureza (g) & $5.1 \pm 1.1$ & $4.7 \pm 0.8$ & $15.6 \pm 0.5$ & $15.2 \pm 0.3$ & $17.6 \pm 0.8$ & $17.3 \pm 1.7$ \\
Fenoles (mg/g) & $42.0 \pm 12.1$ & $39.5 \pm 10.3$ & $22.3 \pm 11.9$ & $20.1 \pm 10.1$ & $17.9 \pm 11.1$ & $17.4 \pm 9.4$ \\
\hline
\end{tabular}

El contenido de agua arrojó diferencias significativas entre épocas $\left(F_{2,114}=273.6 ; P\right.$ $<0.001)$ y entre sitios $\left(F_{1,114}=12.5 ; P<0.001\right)$, el porcentaje de contenido de agua en el Sitio 1 (La Libertad) fue de 34.7\% al inicio de la época lluviosa, $26.1 \%$ al final de la misma y $21.3 \%$ para la época seca (Cuadro 3). El Sitio 2 (Alta Cima) mostró menor porcentaje de agua foliar para cada época con $30.5 \%, 21.8 \%$ y $17 \%$, respectivamente. La interacción sitio-época también mostró diferencia significativa $\left(F_{2,114}=59.02 ; P<0.001\right)$. Al correlacionar el contenido de agua foliar con la herbivoría se obtuvo una correlación positiva y significativa para el inicio de lluvias $\left(r^{2}=0.84 ; P<0.001\right)$ y para el final de la época lluviosa $\left(r^{2}=0.77 ; P<0.001\right)$, no fue detectada significancia entre la herbivoría y el contenido de agua para la época seca (Cuadro 4). 
Cuadro 4. Correlación $\left(r^{2}\right)$ entre la herbivoría de Chamaedorea radicalis (\% área foliar consumida) y los parámetros fisicoquímicos foliares analizados.

\begin{tabular}{lccc}
\hline Parámetros foliares & Inicio de época lluviosa & Final de época lluviosa & Época seca \\
\hline Agua (\%) & $0.84^{* *}$ & $0.77^{* *}$ & 0.21 \\
Nitrógeno foliar (\%) & $0.80^{* *}$ & 0.33 & 0.24 \\
Dureza (g) & -0.36 & $-0.72^{* *}$ & $-0.85^{* *}$ \\
Fenoles (mg/g) & 0.17 & 0.14 & 0.16 \\
\hline
\end{tabular}

$* *=P<0.001$

El contenido de nitrógeno foliar resultó mayor al inicio de la época lluviosa para ambos sitios $(3.9 \% \pm 1.1$ y $3.5 \pm 0.8)$, reduciéndose a medida que la lluvia decrecía hasta la época seca $(1.9 \pm 0.9$ y $1.6 \pm 0.1$, para los Sitios 1 y 2 , respectivamente (Cuadro 3). Las diferencias entre épocas fueron significativas $\left(F_{2,54}=43.1 ; P<\right.$ 0.001) pero no entre sitios, ni en la interacción sitio-época. Este parámetro resultó positivamente correlacionado con la herbivoría solo para la época lluviosa $\left(r^{2}=0.80\right.$; $P<0.001)$ (Cuadro 4).

El contenido de fenoles totales fue mayor en la época lluviosa con $42.0 \pm$ $12.1 \mathrm{mg} / \mathrm{g}$ de peso seco para el Sitio 1 y de $39.5 \pm 12.1 \mathrm{mg} / \mathrm{g}$ de peso seco para el Sitio 2. Este parámetro decreció al paso de las épocas para ambos sitios, el Sitio 1 decreció 1.8 veces para el final de lluvias y 1.2 veces más para la época seca (Cuadro 3). Para el Sitio 2 resultó similar, con 1.9 y 1.1 veces menos concentración fenólica para el fin de la época lluviosa y época seca, respectivamente. Esto fue significativo entre épocas con $F_{2,54}=50.13 ; P<0.001$, pero no entre sitios ni la interacción sitio-época. El contenido fenólico no se correlacionó con la herbivoría en ninguna época analizada (Cuadro 4).

\section{DISCUSIÓN}

Las plantas exhiben tipos diferenciales de defensa contra el ataque de los herbívoros, incluyendo defensas desde las constitutivas hasta la producción de compuestos químicos estructurales (metabolitos secundarios), derivados del metabolismo primario. Esos diferentes tipos de defensa pueden presentarse simultáneamente en las mismas especies vegetales, genotipos, o individuos (Koricheva 2002). Pareciera que las plantas raras veces adoptan por un solo mecanismo de defensa, o una sola toxina (Schoonhoven 1982); es más común que ocurran diversas formas conjuntas de defensa (Paul \& Hay 1986; Hartmann \& Dierich 1998; Romeo 1998). Poseer diversos mecanismos de defensa puede resultar costoso en términos metabólicos para 
la planta, ya que esto se puede reflejar en una reducción en la disponibilidad de recursos para crecer o reproducirse (Koricheva 2002; Strauss et al. 2002).

Varios autores han sugerido la existencia de ciertas restricciones en la asignación de recursos, que actúan de forma simultánea a otras estrategias defensivas, esto promoviendo algunas asociaciones negativas entre los diferentes tipos de defensas. Tales restricciones o concesiones se producen entre los distintos tipos de defensas químicas (Lebreton 1982), entre las defensas químicas y mecánicas (Steward \& Keeler 1988), y entre las defensas constitutiva e inducidas (Mattson et al. 1988).

La sobrecompensación entre las defensas antiherbívoro también son inherentes a la mayoría de las teorías de la defensa de las plantas, tales como la Teoría de la Apariencia (Feeny 1976), la Teoría de la Defensa Óptima (Rhoades 1979), la Hipótesis de la Disponibilidad de Recursos (Coley et al. 1985), y la Hipótesis del crecimiento diferencial propuesta por Herms \& Mattson (1992).

Los resultados de esta investigación sugieren que, independientemente del tipo de vegetación, $C$. radicalis constituye una parte importante del sotobosque. En cuanto al daño foliar, el mayor registro visual fue atribuíble al grupo de los insectos herbívoros. Este hallazgo concuerda con lo expuesto con anterioridad por Oyama \& Dirzo (1991), donde los autores identificaron ocho especies del crisomélido Calyptocephala spp., como los principales herbívoros asociados latitudinalmente a la distribución geográfica de las especies de Chamaedorea spp., a través de un gradiente desde Brasil hasta México.

La herbivoría analizada resultó significativamente diferente entre las épocas y entre los sitios. La densidad de plantas fue mayor en el Sitio 1 (bosque tropical subcaducifolio), con plantas de mayor altura y diámetro, aunque también este sitio presentó la mayor tasa de consumo foliar. Este hecho apunta a favor de la Teoría de la Apariencia propuesta por Feeny (1976) y Rhoades \& Cates (1976), la cual predice que las plantas con alta apariencia en espacio o tiempo invierten mayores recursos en la defensa y poseen defensas químicas "cuantitativas" (principalmente polímeros, e.g. taninos) que reducen su valor nutritivo, son efectivas en grandes concentraciones y son difíciles de superar evolutivamente. Las plantas que poseen menor apariencia invierten menos en defensa y poseen defensas "cualitativas" (monómeros como alcaloides o glucosinolatos), los cuales son disuasivos o tóxicos para casi todos los herbívoros en concentraciones bajas, excepto para unos pocos que las han superado evolutivamente y pueden atacarlas. Este modelo ha sido severamente cuestionado, y se considera que lo que se puede rescatar de él es el concepto de apariencia (Espinosa-García 2001; Fox 1981; Langenheim 1994).

El efecto de la época en la acumulación de herbivoría resultó mayor en el bosque tropical subcaducifolio. Aunque no existen estudios previos, que permitan establecer comparaciones sobre tasas de herbivoría en $C$. radicalis, los resultados obtenidos indican que la herbivoría promedio $\left(0.13 \%\right.$ día- $\left.{ }^{-1}\right)$ son similares a los reportados 
(utilizando el mismo método de medición) a partir de dos especies de árboles tropicales de dosel de Barro Colorado, Panamá: Miconia argentea (Melastomataceae) con $0.15 \%$, Zuelania guidonia (Flacourtiaceae), con $0.14 \%$, superior a Faramea occidentalis (Rubiaceae) con $0.064 \%$, Poulsenia armata (Moraceae) con $0.021 \%$, y menores a 38 especies como Spondias radkolferi (Anacardiaceae) con un 1.5\%, Swartzia simplex (Caesalpinioideae) con 2.5\%, multiflora Guarea (Meliaceae), con $1.18 \%$ y Cecropia obtusifolia (Moraceae) con 0.54\% (Coley 1983). Al comparar nuestros resultados, con otro estudio de la misma región (bosque mesófilo de la Reserva de la Biosfera El Cielo), se aprecia lo siguiente: el promedio diario de herbivoría $C$. radicalis es similar a tres especies de árboles tolerantes (Quercus germana: Fagaceae; Clethra pringlei: Clethraceae; Podocarpus reichei: Podocarpaceae) durante el inicio de estación lluviosa $(0.18 \%)$, al final de la temporada de lluvia $(0.14 \%)$ y, durante la temporada seca $(0.01 \%)$. Sin embargo, son menores a tres especies árboles pioneros (Liquidambar styraciflua: Hammamelidaceae; Cercis canadensis var. mexicana: Fabaceae y Perymenium ovalifolium: Asteraceae) donde estas especies mostraron 3.0 veces menor herbivoría al inicio de la época lluviosa $(0.46 \%), c a .2 .0$ veces al finalizar las lluvias $(0.23 \%)$, y 1.6 veces menos herbivoría en la época seca $(0,15 \%)$ (Sánchez-Ramos 2002).

El daño foliar total (herbivoría anual acumulada) expresado por $C$. radicalis fue de 23.2\% para el Sitio 1 (bosque tropical caducifolio) y de $20.2 \%$ para el Sitio 2 (bosque mixto templado). Este resultado es inferior a la reportado por Carvalho et al. (1999) en Brasil para la palma Euterpe edulis (Arecaceae), donde los autores encontraron que el daño acumulado anual para esta especie fue de 30.3\%. Sin embargo, esto se encuentra dentro del porcentaje que tolera otra especie del mismo género $(C$. tepejilote) en la región de Los Tuxtlas, Veracruz donde Oyama \& Mendoza (1990) llevaron a cabo un estudio de defoliación artificial en esta especie, encontrando que con $25.0 \%$ de defoliación la planta es capaz de producir el doble de follaje.

La dureza foliar se incrementó directamente proporcional al avance de las épocas del año. Este hecho concuerda con otros estudios como el de Filip et al. (1995), donde los autores midieron la dureza foliar de 16 especies tropicales en Chamela, Jalisco. Otro estudio similar es el de Coley (1983), donde la autora analizó la dureza foliar de 46 especies tropicales en Barro Colorado, Panamá y un último estudio fue el conducido por Sánchez-Ramos (2002), con 46 especies propias del bosque mesófilo de la Reserva El Cielo. En todos estos estudios el método de medición de la dureza fue el mismo (véase Feeny 1970 y Dirzo 1984).

Al progresar la época del año la planta lignifica sus tejidos, así que como era de esperarse, la dureza foliar se correlacionó negativamente con la herbivoría, de modo que a medida que aumentó la dureza de hoja, el consumo foliar disminuyó. Sin embargo, el agua y el contenido de nitrógeno se correlacionaron positivamente con la herbivoría, y sorprendentemente la concentración de compuestos fenólicos no 
resultó un efectivo disuasivo del ataque de herbívoros en esta especie. Nuestro estudio demuestra que la dureza foliar constituyó la defensa más eficaz contra los herbívoros.

Aunque el Sitio 1 (bosque tropical subcaducifolio), se muestra un promedio de lluvia mayor, el contenido de agua foliar fue mayor en el Sitio 2 (bosque mixto templado). Esto puede estar directamente relacionado con la disponibilidad de este recurso durante todo el año a través de la presencia de nubes (lluvia horizontal), ya que es una zona de transición con el bosque mesófilo de montaña o bosque de nubes, donde este tipo de lluvia significa el 60\% del total de la humedad de este ecosistema (Webster 1995).

Otras características de las hojas, diferentes a la concentración de defensas químicas, el contenido de nutrientes (i.e., nitrógeno foliar), el contenido de agua foliar y la dureza de la hoja pueden ser afectadas por la disponibilidad de la luz y, potencialmente las variaciones en la palatabilidad de las hojas (Bowers \& Porter 1981; Waller 1982; Berisha 1986). Sin embargo, la mayoría de los estudios han demostrado que la principal causa de la disminución de los niveles de daño foliar es la concentración de defensas químicas, que normalmente aumenta con la disponibilidad de luz (Crawley 1983; Coley et al. 1985; Price et al. 1989; Precio 1997). Sin embargo, nuestro estudio con $C$. radicalis difiere de estas observaciones, señalando como la mejor defensa la dureza de la hoja.

En conclusión, una estrategia defensiva (contenido de fenoles totales) contra la herbivoría que resulta exitosa para otras especies, no parece desempeñar un papel significativo en la palma camedor. Sin embargo, la mayor amenaza para la palmilla no es el daño por herbívoros. Se trata de la extracción comercial de la población local para su comercialización en todo el mundo. Existe presión extractiva en la especie, debido a que el follaje cosechado debe estar libre de daño y manchado foliar. Otro factor que desfavorece a la especie, es que las hojas deben ser cosechadas enteras cuando en promedio una planta tiene sólo tres. Además de que los insectos eliminan ca. $20.0 \%$ del follaje anualmente, los seres humanos eliminan ca. $40.0 \%$ (número total de hojas/número de hojas cortadas). Por esta razón, los insectos herbívoros pueden beneficiar a $C$. radicalis, ya que interfiere con el creciente proceso extractivo de la especie. En la Reserva de la Biosfera El Cielo, poder encontrar plantas que no han sido cortadas es difícil. Por lo tanto, los insectos herbívoros de $C$. radicalis pueden ser un aspecto importante que impide que esta planta amenazada llegue a estar en peligro de extinción. Estudios posteriores, que versen sobre el efecto de la herbivoría simulada y sus consecuencias ecológicas, dará una mejor comprensión de la historia natural de esta importante especie.

AGRADECIMIENTOS. Los autores agradecen al proyecto FOMIX (TAMS-2002-CO1-3536 (asignado a G.S.R) por el financiamiento de estudio. Agradecemos también a Sherman A. Phillips Jr. del Colegio de Ciencias Agrícolas y Recursos Naturales del Texas Tech University. 


\section{LITERATURA CITADA}

Bazzaz, F. A. Chiariello, N. R., Coley, P. D. \& L.F. Pitelka. 1987. Allocating resources to reproduction and defense. Bioscience, 37: 58-67.

Bell, E.A. 1974. Control Plant Insects. Institute of Biology. University of California. 403 pp.

Berish, C. W. 1986. Leaf-cutting ants (Atta cephalotes) select nitrogen-rich foliage. American Midland Naturalist, 115: 267-276.

Bowers, M. A. \& S. D. Porter. 1981. Effect of foraging distance on water content of substrates harvested by Atta colombica. Ecology, 74: 273-275.

Carvalho, R. M., F. R. Martins \& F. A. Santos. 1999. Leaf Ecology of Pre-reproductive Ontogenetic Stages of the Palm Tree Euterpe edulis Mart. (Arecaceae). Annals of Botany, 83: 225-233.

Coley, P.D. 1981. Ecological and evolutionary responses of tropical trees to herbivory: a quantitative analysis of grazing damage, plant defenses, and growth rates. University of Chicago, Chicago, Illinois. $130 \mathrm{pp}$.

Coley, P. D. 1983. Herbivory and defensive characteristics of tree species in a lowland tropical forest. Ecological Monographs, 53: 209-33.

Coley, P. D. 1990. Tasas de herbivorismo en diferentes árboles tropicales. Pp. 191-200. In: Leigh, E. G. Jr.; R. A. Stanley, D. M. Windsor, (Eds.). Ecología de un bosque tropical. The Smithsonian Tropical Research Institute, Balboa, Panamá.

Coley, P.D. \& T. M. Aide 1991. Comparison of herbivory and plant defenses in temperate and tropical broad-leaved 358 I. J. Wright \& K. Cannon 2001 British Ecological Society, Functional Ecology, 15, 351-359 forests. Plant-Animal Interactions: Evolutionary Ecology in Tropical and Temperate Regions (O. W. Price, T. M. Lewinsohn, G. W. Fernandes \& W. W. Benson, Eds.), pp. 25-49. Wiley, New York.

Coley, P. D., J. P. Bryant, \& F. S. Chapin III. 1985. Resource availability and plant antiherbivore defenses. Science, 230: 895-899.

Crankshaw, D.R. \& J.H. Langenheim. 1981. Variation in terpenes and phenolics through leaf development in Hymenaea and its possible significance to herbivory. Biochemistry System Ecology, 9: 115-124.

Crawley, M. J. 1983. Herbivory. University of California Press, Berkeley.

Deverall, B.J. 1977. Defense Mechanisms of Plants. Cambridge University Press, London and New York. 240 pp.

Dirzo, R. 1984. Herbivory: a phytocentric overview. Pp. 141-165. In: Dirzo, R. y J. Sarukhán (Eds.) Perspectives in plant population ecology. Sinauer Associates Publishers. Sunderland. Massachussets.

Dirzo, R. 1986. Insectos y plantas: protagonistas de una historia de la vida. Secretaría de Educación Pública. Dirección General de Publicaciones y Medios. 1ª Edición. México, D.F. pp. 35-37.

Dirzo, R. 1987. Estudios sobre interacciones planta-herbívoro en Los Tuxtlas, Veracruz. In: Clark, D. A., R. Dirzo, N. Fetcher (Eds.). Ecología y ecofisiología de plantas en los bosques mesoamericanos. Revista de Biología Tropical, 35: 119-131.

Dirzo, R. \& C.A. Domínguez. 1995. Plant-herbivore interactions in Mesoamerican tropical dry forest. Pp. 304-325. In: Bullock, S.H.; H.A. Mooney and E. Medina (Eds.). Seasonally Dry Tropical Forest. Cambridge University Press.

Ehrlich, P.R. \& P.H. Raven. 1964. Butterfiles and plants: a study in co-evolution. Evolution, 18: 586608.

Espinosa-García F. J. 2001. La diversidad de los metabolitos secundarios y la teoría de la defensa vegetal. Pp. 231-250. In: Anaya, A.L., F.J.G. Espinosa y R.C. Ortega (Eds.). Interacciones químicas entre organismos. Aspectos básicos y perspectivas de su aplicación. Universidad Nacional Autónoma de México. México, D.F. 
Feeny, P. 1970. Seasonal changes in oak leaf tannins and nutrients as a cause of spring feeding by winter moth caterpillars. Ecology, 51: 565-581.

Feeny, P. 1976. Plant apparency and chemical defense. In: Wallace J, R.L. Mansell (Eds.). Chemical interactions between plants and insects. Recent Advances of Phytochemistry, 10: 1-40.

Filip, V., R. Dirzo, J. M. Maass \& J. K. Sarukhán. 1995. Within- and among-year variation in the levels of herbivory on the foliage of trees from a Mexican tropical deciduous forest. Biotropica, 27: 78-86.

Fox, L.R. 1981. Defense and dynamics in plant - herbivore systems. American Zoologist, 21: 853-864.

Gentry, A. 1982. Neotropical floristic diversity: Phytogeographical connections between Central and South America. Pleistocene climatic fluctuations or an accident of the Andean orogeny? Annual Missouri Botanical Garden, 69: 557-593.

Gershenzon, J. 1984. Changes in the Level of Plant Secondary Metabolites Under Water and Nutrient Stress. Pp. 273-320. In: B. Timmermann, C. Steelink y F. Loewus (Eds.). Phytochemical Adaptatnions to Stress. Plenum Press, Nueva York.

González M. J. M., G. Sánchez-Ramos, F.E. Briones \& M.A.Bortoni. 2003. Variación de la herbivoría, contenido de nitrógeno y fenoles totales foliares en palma camedor (Chamaedorea radicalis Mart.) en una localidad de Gómez Farías, Tamaulipas. BIOTAM (Nueva Serie), 14(3): 27-46.

Harborne, J.B. 1988. Introduction to ecological biochemistry. 3d. edition. London Academic Press. $320 \mathrm{pp}$.

Harper, J. L. 1977. Population biology of plants. Academic Press, New York.

Hartmann, T., \& B. Dierich. 1998. Chemical diversity and variation of pyrrolizidine alkaloids of the senecionine type: biological need or coincidence? Planta, 206: 443-451.

Henley, R. W., A. R. Chase, \& L. S. Osborne. 2000. Chamaedorea palm production guide. (on line: http://gnv.ifas.ufl.edu/ apkweb/folnotes/chamaed.htm

Herms, D. A., \& W. J. Mattson. 1992. The dilemma of plants: to grow or defend. Quarterly Review of Biology, 67: 283-335.

Hodel, D.R. 1992. Chamaedorea Palms. The International Palm Society. Allen Press, Lawrence.

Hochuli, D.F. 1996. The ecology of plant/insect interactions-implications of digestive strategy for feeding by phytophagous insects. Oikos, 75: 133-141.

Hunter, M. D. \& Schultz, J. C. 1995. Fertilization mitigates chemical induction and herbivore responses within damaged oak trees. Ecology, 76: 1226-1232.

Janzen, D.H. 1981. Patterns of herbivory in a tropical deciduous forest. Biotropica, 13: 271-282.

Janzen, D.H. \& P.G. Waterman. 1984. A seasonal census of phenolics, fiber and alkaloids in foliage of forest trees in Costa Rica: some factors influencing their distribution and relation to host selection by Sphingidae and Saturniidae. Biological Journal of Linnean Society, 21: 439-454.

Karban, R. \& Baldwin, I. T. 1997. Induced responses to herbivory. University of Chicago Press, Chicago.

Koricheva, J. 2002. The carbon-nutrient balance hypothesis is dead; long live the carbon-nutrient balance hypotheses? Oikos, 98: 537-539.

Langenheim, J.H. 1994. Higher plant terpenoids: a phytocentric overview of their ecological roles. Journal of Chemical Ecology, 20: 1223-1280.

Lebreton, P. 1982. Tanins ou alkaloïdes: deux tactiques phytochimiques de dissuasion des herbivores. Revue d'Ecologie la Terre et la Vie, 36: 539-572.

Marquis, R.J. 1984. Leaf herbivores decrease fitness of a tropical plant. Science, 226: 537-539.

Marquis RJ. 1987. Variación en la herbivoría foliar y su importancia selectiva en Piper arieianum (Piperaceae). Revista de Biología Tropical, 35: 133-150. 
Mattson, W. J., R. K. Lawrence, R. A. Haack, D. A. Herms, \& P. J. Charles. 1988. Defensive strategies of woody plants against different insect-feeding guilds in relation to plant ecological strategies and intimacy of association with insects. Pp. 3-38 In: W. J. Mattson, J. Levieux, and C. Bernard-Dagan (Eds.). Mechanisms of woody plant defenses against insects. Springer, New York.

McNaughton, S. J. 1983. Compensatory plant growth as a response to herbivory. Oikos, 40: 329-336.

Mora-Olivo, A., J. L. L. Mora, J. L. P. Jiménez \% J. S. Sifuentes. 1997. Vegetación y flora asociada a la palmilla (Chamaedorea radicalis Mart.) en la Reserva de la Biosfera El Cielo. BIOTAM, 8: 110.

Oyama, K. 1987. Demografía y dinámica poblacional de Chamaedorea tepejilote Liebm. en la selva de Los Tuxtlas, Veracruz (México). Tesis de Maestría, inédita. Facultad de Ciencias UNAM, México.

Oyama, K. \& R. Dirzo. 1991. Ecological aspects on the interaction between Chamaedorea tepejilote, a dioecious palm and Calyptocephala marginipennis, an herbivorous beetle, in a Mexican rain forest. Principes, 35: 86-93.

Oyama, K., \& A. Mendoza. 1990. Effects of defoliation on growth, reproduction, and survival of a Neotropical dioecious palm, Chamaedorea tepejilote. Biotropica, 22: 119-123.

Paul, V. J., \& M. E. Hay. 1986. Seaweed susceptibility to herbivory: chemical and morphological correlates. Marine Ecology Progress Series, 33: 255-264.

Ponce-Vargas, A. I. Luna-Vega, O. Alcántara-Ayala \& C. A. Ruiz-Jiménez. 2006. Florística del bosque mesófilo de montaña de Monte Grande, Lolotla, Hidalgo, México. Revista Mexicana de Biodiversidad, 77: 177-190.

Price, P. 1997. Insect ecology. 3rd ed. John Wiley \& Sons. Nueva York.

Price, P., G Waring, R Julkunen-Titto, J. Tahvanainen, H. Mooney \& T. Craig. 1989. The carbonnutrient balance hypothesis in within-species phytochemical variation of Salix lasiolepsis. Journal Chemical Ecology, 15: 1117-1131.

Quero, H. J. 1989. Flora genérica de las Arecáceas de México. Tesis Doctoral, inédita. Facultad de Ciencias, Universidad Nacional Autónoma de México, México.

Rhoades, D. F. 1979. Evolution of plant chemical defense against herbivores. Pp. 3-54. In: G. A. Rosenthal and D. H. Janzen (Eds.). Herbivores: their interaction with secondary plant metabolites. Academic Press, New York.

Rhoades, D.F. \& R.G. Cates. 1976. Toward a general theory of plant herbivore chemestry. Pp. 168213. In: J. Wallace \& R.L. Mansell (Eds.). Biochemical interactions between plants and insects. Recent advances in Phytochemistry. Plenum Press. New York, New York. USA.

Romeo, J. T. 1998. Functional multiplicity among nonprotein amino acids in Mimosoid legumes: a case against redundancy. Ecoscience, 5:287-294.

Sánchez-Ramos, G. 2002. Patrones de interacción planta-herbívoro en el bosque mesófilo de Gómez Farías, Tamaulipas, México. Tesis Doctoral, inédita. Facultad de Ciencias, Universidad Nacional Autónoma de México, México, D.F.

Sánchez-Ramos, G, R. Dirzo, \& M. A. Balcázar-Lara. 1999. Especificidad y herbivoría de Lepidoptera sobre especies pioneras y tolerantes del bosque mesófilo de la Reserva de la Biosfera El Cielo, Tamaulipas, México. Acta Zoológica Mexicana (n.s.), 78: 103-118.

Sánchez-Ramos, G. \& P. Reyes-Castillo. 2006. Ecological interaction of Pantophthalmus roseni (Enderlein) (Diptera: Pantophthalmidae) and the Red Oak Quercus germana Sachltdl. Et Cham. (Fagaceae) in a Mexican Cloud Forest. Acta Zoológica Mexicana (n.s.), 22 (2): 45-56.

Schoonhoven, L. M. 1982. Biological aspects of antifeedants. Entomologia Experimentalis et Applicata, 31: 57-69.

Schultz, J.C. \& I.T. Baldwin. 1982. Oak leaf quality declines in response to defoliation by gypsy moth larvae. Science, 217: 149-151. 
Sánchez-Ramos et al.: Estudio de la herbivoría de Chamaedorea radicalis

Steward, J. L., \& K. H. Keeler. 1988. Are there trade-offs among antiherbivore defenses in Ipomoea (Convolvulaceae)? Oikos, 53: 79-86.

Strauss, S. Y., J. A. Rudgers, J. A. Lau, \& R. E. Irwin. 2002. Direct and ecological costs of resistance to herbivory. Trends in Ecology \& Evolution, 17: 278-285.

Technicon Industrial Systems. 1977. Technicon Industrial Method No. 329-74 W/B. Individual/simultaneous determinations of nitrogen and/or phosphorus in BD acid digests: Technicon Ind. Syst., Tarrytown.

Tiffin, P. 2000. Mechanisms of tolerance to herbivore damage: what do we know? Evolutionary Ecology, 14 (5): 523-536.

Trejo, H. L. 1992. La palmilla (Chamaedorea spp.) un recurso forestal no maderable en la Reserva de la Biosfera El Cielo. Revista de la Universidad Autónoma de Tamaulipas, 21: 53-55.

Trumble, J. T., Kolodny-Hirsch, D. M. \& Ting, I. P. 1993. Plant compensation for arthropod herbivory. Annual Review of Entomology, 38: 93-119.

Turner, N. C. 1981. Techniques and experimental approaches for the measurements of plant water status. Journal of Plant Soil Science, 58:339-366.

Vovides, A. P. 1981. Lista preliminar de plantas mexicanas raras o en peligro de extinción. Biótica, 6 : 219-228.

Waller, D. A. 1982. Leaf-cutting ants and live oak: the role of leaf toughness in seasonal and intraspecific host choice. Entomologia Experimentalis et Applicata, 32:146-150.

Waterman, P. G. J. A. M. Ross, \& D. McKey. 1984. Factors affecting levels of some phenolic compounds, digestibility, and nitrogen content of the mature leaves of Barteria fistulosa (Passifloraceae). Journal of Chemical Ecology, 10: 387-401.

Waterman, P. G., \& S. Mole. 1994. Analysis of phenolic plant metabolites. Blackwell Scientific Publications, London.

Webster. G. L. 1995. The Panorama of neotropical cloud forests, pp. 53-77. In: Churchill. S. P., H. Balslev, E. Forero \& J. L. Luteyn (Eds.). Biodiversity and conservation of neotropical montane forest. The New York botanical Garden. Nueva York.

Whitman, T.G. \& S. Mooper. 1985. Chronic herbivory: Impacts on architecture and sex expression of pynion pine. Science, 228: 1089-1091.

Whittaker, R.H., \& P.P. Feeny. 1971. Allelochemics: chemical interactions between species. Science, 171: $757-770$.

Wofle, L.M. 1997. Flower herbivory and agall formation on males and females of Neea psychotroides, a dioecious tree. Biotropica, 29: 169.

Zucker, W.V. 1983. Tannins: does structure determine function? An ecological perspective. American Naturalist, 121: 335-365.

Zar, J. H. 1984. Biostatistical analysis. Prentice Hall, Inc., N.J. 